\title{
Findings About Events or Interventions Sponsor Defined Identifier
}

National Cancer Institute

\section{Source}

National Cancer Institute. Findings About Events or Interventions Sponsor Defined

Identifier. NCI Thesaurus. Code C87890.

One or more sponsor defined characters used to identify, name, or characterize the finding. 